\title{
Perturbative quark bound states in pNRQCD*
}

\author{
Nora Brambilla $a^{\dagger}$ \\ ${ }^{a}$ Institut für Theoretische Physik, Universität Heidelberg \\ Philosophenweg 16, D-69120 Heidelberg, Germany \\ email:n.brambilla@thphys.uni-heidelberg.de
}

\begin{abstract}
In the framework of the QCD effective field theory called potential Non-Relativistic QCD, we explore quarkantiquark bound systems that may be dominated by the perturbative interaction and we discuss the extent of validity of such a picture. Some phenomenological implications are outlined.
\end{abstract}

\section{INTRODUCTION}

If we have a chance to understand the QCD bound-state dynamics without resorting to the lattice or to models [1], this is likely to be in the cases of bound systems whose interaction is dominated by the perturbative dynamics. In such cases non-perturbative corrections exist but are small and can be parameterized in terms of local or non-local condensates. In the following I will call such systems 'Coulombic' or 'quasiCoulombic'. These situations are particularly interesting not only because, as I will show, they have phenomenological relevance, but especially because they allow us to understand much more about QCD. Here, I will exclusively address such situations.

Typically, systems that may be 'Coulombic or quasi' are bound states composed only by heavy quarks (and gluons). In such cases, at least for the lowest states, the characteristic radius $\langle r\rangle(r$ being the $q \bar{q}$ distance) of the system is quite small and this intuitively justifies the idea that they are dominated by the perturbative dynamics: the system is too small to probe the confinement effects, which arise at a scale $1 / \Lambda_{\mathrm{QCD}} \gg\langle r\rangle$.

Here, I will introduce an effective field theory called potential Non-Relativistic QCD (pNRQCD)[2, 3], that clarifies such picture and allows us to include systematically perturbative

${ }^{*}$ Invited talk given at the International Euroconference QCD 00, Montpellier, France, July 6-13th , 2000. To be published in Nucl. Phys. Proc. Suppl.

${ }^{\dagger}$ Alexander von Humboldt fellow and non-perturbative contributions. Since such effective theory is constructed to be completely equivalent to $\mathrm{QCD}$, all the information that we will obtain go to deepen our knowledge of bound systems in QCD. For the non-perturbative situation see the talk by A. Vairo at this Conference [4] and [3,5].

\section{THE SCALES of QUARKONIUM}

As it is apparent from the spectra, heavy quarkonia are non-relativistic systems. Thus, they may be described in first approximation using a Schrödinger equation with a potential interaction. This amounts to saying that the heavy quark bound state is characterized by three energy scales, hierarchically ordered by the quark velocity $v \ll 1$ : the quark mass $m$ (hard scale), the momentum $m v$ (soft scale (S)), and the binding energy $m v^{2}$ (ultrasoft scale (US)). In the Coulombic or quasi-Coulombic situation it is $v \sim$ $\alpha_{\mathrm{s}}$.

In perturbative calculations, these scales typically get mixed in the Feynman diagrams and originate technical complications (the same happens in QED, e.g. for positronium).

In QCD a further conceptual complication arises if we take into account the existence of the non-perturbative scale $\Lambda_{\mathrm{QCD}}$, at which the nonperturbative effects become dominant. For heavy quarks only the hard scale $m$ is surely bigger than $\Lambda_{\mathrm{QCD}}$ and can be treated perturbatively.

The existence of these different scales makes even a purely perturbative definition of the static 


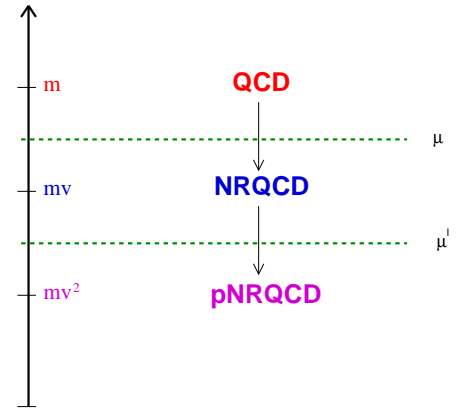

Figure 1. Scales and EFTs in NR bound state systems.

$q \bar{q}$ potential not free from complications. Let us consider the energy of the static quark sources, $E_{s}(r) \equiv \lim _{T \rightarrow \infty} \frac{i}{T} \ln \left\langle W_{\square}\right\rangle$, (being $W_{\square}$ the static Wilson loop of size $\mathbf{r} \times T$, and the symbol \langle\rangle being the average over the gauge fields), which is usually considered as a definition of the static potential. At three loops $E_{s}$ shows infrared divergences 6, [7]. These singularities may indeed be regulated, upon resummation of a certain class of diagrams, which give rise to a sort of dynamical cut-off provided by the difference between the singlet and the octet potential. However, such a dynamical scale is of the same order of the kinetic energy for quarks of large but finite mass and, therefore, should not be included into a proper definition of the static potential in the sense of the Schrödinger equation. This is similar to what happens for the Lamb shift in QED at order $1 / m^{2}$. In QCD this effect calls, even in the definition of the static potential, for a rigorous treatment of the bound-state scales.

To address the multiscale dynamics of the heavy quark bound state, the concept of effective field theory turns out to be not only helpful but actually necessary. QCD effective field theories (EFT) with less and less degrees of freedom, can be obtained by systematically integrating out the scales above the energy we aim to describe. This procedure leads ultimately to a field theory derived quantum mechanical description of these systems. The corresponding EFT is called pNRQCD [2,3] (cf.also[8]). Here, all the dynamical regimes are organized in a systematic expansion and the above mentioned problems are solved. In particular we get a procedure to calculate the static potential beyond two loops and to obtain the energy levels beyond next-to-next-to leading order $\left(\mathrm{N}^{2} \mathrm{LO}\right)$ 月.

\section{NRQCD and pNRQCD}

Since the typical scales of the system are widely separated, it is possible to perform an expansion of one scale in terms of the others and integrate out step by step the scales at higher energy. Roughly speaking, first one writes the EFT as a $1 / m$ expansion and integrates out the scale $m$ in QCD: this leads to NRQCD. Later, one writes the EFT as an expansion in the inverse of the soft scale, the so called multipole expansion, and integrates out the soft scale in NRQCD: this leads to pNRQCD. See Fig. 1. The effective theory supplies us with the procedure to make this expansion consistent with the ultraviolet behaviour of QCD and consistent with a systematic power counting in the small expansion parameter $v$. Only if the physical system is such that the scales we are integrating out are much bigger than $\Lambda_{\mathrm{QCD}}$, namely $m v \gg \Lambda_{\mathrm{QCD}}$, then it is possible to take advantage of the further simplification of performing the calculations in a perturbative expansion in $\alpha_{s}$ and to recover a perturbative description of the dynamics. This is the situation we consider here.

The Lagrangian of NRQCD [9] can be organized in powers of $1 / \mathrm{m}$. In the two-fermions sector it is of the type obtained via a Foldy-Wouthuysen transformation, but, since we are modifying the ultraviolet behaviour of the theory, matching coefficients and new operators have to be added in order to mock up the effects of heavy particles and high energy modes into the low energy EFT. Since the scale of the mass of the heavy quark is perturbative, the scale $\mu$ of the matching from QCD to NRQCD, $m v<\mu<m$, lies also in the perturbative regime. The integration of degrees of freedom is done in practice with a matching

\footnotetext{
${ }^{3}$ The non-relativistic limit described by the Schrödinger equation with the static potential is called 'leading order' (LO); contributions corresponding to corrections of order $v^{n}$ to this limit are called $\mathrm{N}^{n} \mathrm{LO}$. LL means 'leading log'.
} 
procedure i.e. by comparing on shell amplitudes, order by order in $1 / m$ and in $\alpha_{s}$, in QCD and in NRQCD. The difference is encoded into the matching coefficients that typically depend nonanalytically on the scale $m$ which has been integrated out: $c \simeq A \alpha_{s}\left(\ln \frac{m}{\mu}+B\right)$. One works typically in dimensional regularization, $\overline{M S}$ scheme, and with quark pole masses.

After integrating out the soft scale in NRQCD, pNRQCD is obtained. The Lagrangian of $\mathrm{pN}$ RQCD is organized in powers of $1 / m$ and $\mathbf{r}$ (multipole expansion). The matching is done by comparing appropriate off-shell amplitudes in NRQCD and in pNRQCD, order by order in $1 / m$, $\alpha_{s}$ and order by order in the multipole expansion. The matching coefficients are non-analytic functions of $\mathbf{r}$ and have typically the following structure: $V \simeq \mathcal{V}\left(\mathbf{r}, \mathbf{p}, \mathbf{S}_{\mathbf{1}}, \mathbf{S}_{2}\right)\left(A^{\prime} \ln m r+B^{\prime} \ln \mu^{\prime} r+C\right)$.

\section{4. pNRQCD for $m v \gg \Lambda_{\mathrm{QCD}}$}

At the scale of the matching $\mu^{\prime}\left(m v \gg \mu^{\prime} \gg\right.$ $\left.m v^{2}, \Lambda_{\mathrm{QCD}}\right)$ we have still quarks and gluons. We denote by $\mathbf{R} \equiv\left(\mathbf{x}_{1}+\mathbf{x}_{2}\right) / 2$ the center-of-mass of the $q \bar{q}$ system and by $\mathbf{r} \equiv \mathbf{x}_{\mathbf{1}}-\mathbf{x}_{\mathbf{2}}$ the relative distance. The effective degrees of freedom are: $q \bar{q}$ states (that can be decomposed into a singlet $S(\mathbf{R}, \mathbf{r}, t)$ and an octet $O(\mathbf{R}, \mathbf{r}, t)$ under color transformations) with energy of the order of the next relevant scale, $O\left(\Lambda_{Q C D}, m v^{2}\right)$, and momentum ${ }^{4} \mathbf{p}$ of order $O(m v)$, plus ultrasoft gluons $A_{\mu}(\mathbf{R}, t)$ with energy and momentum of order $O\left(\Lambda_{\mathrm{QCD}}, m v^{2}\right)$. Notice that all the gluon fields are multipole expanded. The Lagrangian is then an expansion in the small quantities $p / m, 1 / r m$ and in $O\left(\Lambda_{\mathrm{QCD}}, m v^{2}\right) \times r$. The pNRQCD Lagrangian is given at the leading order in the multipole expansion by[3]:

$$
\begin{aligned}
& \mathcal{L}=\operatorname{Tr}\left\{\mathrm{S}^{\dagger}\left(i \partial_{0}-\frac{\mathbf{p}^{2}}{m}-V_{s}(r)-\sum_{n=1} \frac{V_{s}^{(n)}}{m^{n}}\right) \mathrm{S}\right. \\
& \left.+\mathrm{O}^{\dagger}\left(i D_{0}-\frac{\mathbf{p}^{2}}{m}-V_{o}(r)-\sum_{n=1} \frac{V_{o}^{(n)}}{m^{n}}\right) \mathrm{O}\right\} \\
& +g V_{A}(r) \operatorname{Tr}\left\{\mathrm{O}^{\dagger} \mathbf{r} \cdot \mathbf{E} \mathrm{S}+\mathrm{S}^{\dagger} \mathbf{r} \cdot \mathbf{E} \mathrm{O}\right\}
\end{aligned}
$$

\footnotetext{
${ }^{4}$ Although, for simplicity, we describe the matching between NRQCD and pNRQCD as integrating out the soft scale, the relative momentum $\mathbf{p}$ of the quarks is still soft.
}

\begin{tabular}{|c|c|}
\hline scale & bound states \\
\hline$m$ & $\psi(2 S), \chi_{c 0}(1 P), \ldots$ \\
& $\Upsilon(2 S), \chi_{b 0}(1 P), \ldots$ \\
& long-range hybrids, $\ldots$ \\
$m v$ & $\eta_{c}, J / \psi, B_{c}, \ldots$ \\
& short-range hybrids, $\ldots$ \\
$m v^{2}$ & $t \bar{t}, \Upsilon(1 S), \ldots$ \\
$<m v^{2}$ & QED bound states \\
\hline
\end{tabular}

Table 1

An indicative classification of the states which can be treated perturbatively up to a scale $\mathrm{mv}^{n}$ assuming the criterion $m \psi_{\text {pert }}^{n} / \Lambda_{\mathrm{QCD}} \gg 1$.

$+g \frac{V_{B}(r)}{2} \operatorname{Tr}\left\{\mathrm{O}^{\dagger} \mathbf{r} \cdot \mathbf{E} \mathrm{O}+\mathrm{O}^{\dagger} \mathrm{Or} \cdot \mathbf{E}\right\}-\frac{1}{4} F_{\mu \nu}^{a} F^{\mu \nu a}$.

All the gauge fields in Eq. (11) are evaluated in $\mathbf{R}$ and $t$. In particular $\mathbf{E} \equiv \mathbf{E}(\mathbf{R}, t)$ and $i D_{0} \mathrm{O} \equiv i \partial_{0} \mathrm{O}-g\left[A_{0}(\mathbf{R}, t), \mathrm{O}\right]$. The quantities denoted by $V$ are the matching coefficients. We call $V_{s}$ and $V_{o}$ the singlet and octet static matching potentials respectively. At the leading order in the multipole expansion, the singlet sector of the Lagrangian gives rise to equations of motion of the Schrödinger type. The two last lines of (1) contain (apart from the Yang-Mills Lagrangian) retardation (or non-potential) effects that start at the NLO in the multipole expansion. At this order the non-potential effects come from the singlet-octet and octet-octet interactions mediated by a ultrasoft chromoelectric field.

Various applications of pNRQCD have been presented at previous editions of this Conference[2, 10].

Recalling that $\mathbf{r} \sim 1 / m v$ and that the operators count like the next relevant scale, $O\left(m v^{2}, \Lambda_{\mathrm{QCD}}\right)$, to the power of the dimension, it follows that each term in the pNRQCD Lagrangian has a definite power counting. This feature makes $\mathcal{L}_{\mathrm{pNRQCD}}$ a suitable tool for bound state calculations: being interested in knowing the energy levels up to some power $v^{n}$, we just need to evaluate the contributions of this size from the Lagrangian. From the power counting e.g., it follows that the interaction of quarks with ultrasoft gluons is suppressed in the Lagrangian by $v$ with respect to the LO ( by $g v$ if $m v^{2} \gg \Lambda_{\mathrm{QCD}}$ ).

In particular, pNRQCD provides us with the 
way of obtaining the matching potentials via the matching procedure at any order of the perturbative expansion[3]. In the EFT language the potential is defined upon the integration of all the scales up to the ultrasoft scale $m v^{2}$. From the matching to NRQCD in the situation $\Lambda_{\mathrm{QCD}} \ll$ $m v$ we can easily obtain the matching potential $V_{s}$ at $\mathrm{N}^{3} \mathrm{LL}$ [7]

$V_{s}(r)=\left.E_{s}(r)\right|_{2-\text { loop }+\mathrm{N}^{3} \mathrm{LL}}+C_{F} \frac{\alpha_{\mathrm{s}}}{r} \frac{\alpha_{\mathrm{s}}^{3}}{\pi} \frac{C_{A}^{3}}{12} \ln \frac{C_{A} \alpha_{\mathrm{s}}}{2 r \mu^{\prime}}$,

where $E_{s}$ is the static energy defined in Sec.2 (regulated by the resummation). We note that $V_{s}$ and $E_{s}$ would coincide in QED and that, therefore, this difference here is a genuine QCD feature. Such difference is switched on at NLO in the multipole expansion. An explicit calculation gives [7]

$$
\begin{aligned}
& V_{s}(r) \equiv-C_{F} \frac{\alpha_{V}\left(r, \mu^{\prime}\right)}{r}, \\
& \alpha_{V}(r, \mu)=\alpha_{\mathrm{s}}(r)\left\{1+\left(a_{1}+2 \gamma_{E} \beta_{0}\right) \frac{\alpha_{\mathrm{s}}(r)}{4 \pi}\right. \\
& +\frac{\alpha_{\mathrm{s}}^{2}(r)}{16 \pi^{2}}\left[\gamma_{E}\left(4 a_{1} \beta_{0}+2 \beta_{1}\right)+\left(\frac{\pi^{2}}{3}+4 \gamma_{E}^{2}\right) \beta_{0}^{2}\right. \\
& \left.\left.+a_{2}\right]+\frac{C_{A}^{3}}{12} \frac{\alpha_{\mathrm{s}}^{3}(r)}{\pi} \ln r \mu^{\prime}\right\},
\end{aligned}
$$

where $\beta_{n}$ are the coefficients of the beta function ( $\alpha_{\mathrm{s}}$ is in the $\overline{\mathrm{MS}}$ scheme), and $a_{1}$ and $a_{2}$ were given in [11]. We see that the interpretation of the potentials as matching coefficients in $\mathrm{pN}$ RQCD implies that the Coulomb potential is not simply coincident with the static energy $E_{s}$. The Coulomb potential turns out to be sensitive to the ultrasoft scale but infrared finite. The same happens with the other potentials (like $V_{o}$ or the potentials that bear corrections in $1 / \mathrm{m}^{n}$ ) that can equally be calculated via the matching procedure. The $\mu^{\prime}$ scale dependence in the potential is cancelled for any physical process by the contribution of the ultrasoft gluons that are cutoff at the scale $\mu^{\prime}$.

Then, there are two situations 15]. If $m v \gg$ $m v^{2} \gtrsim \Lambda_{\mathrm{QCD}}$, the system is described up to or$\operatorname{der} \alpha_{\mathrm{s}}^{4}$ by a potential which is entirely accessible to perturbative QCD. Non-potential effects

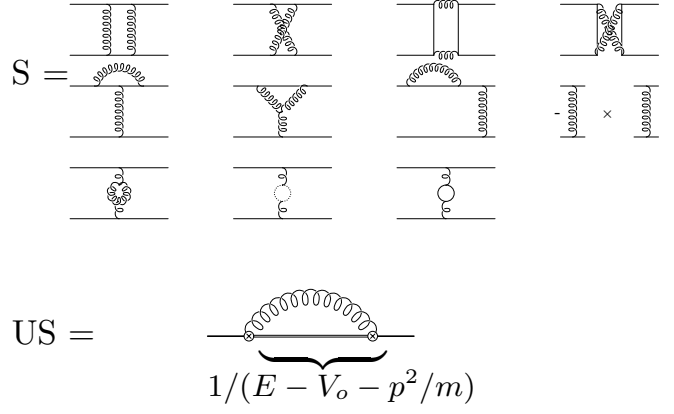

Figure 2. Graphs contributing to the spectrum up to $\mathrm{N}^{3} L L$.

start at order $\alpha_{\mathrm{s}}^{5} \ln \mu^{\prime}$ 16. We call Coulombic this kind of system. Non-perturbative effects are of non-potential type and can be encoded into local (à la Voloshin-Leutwyler 14) or non-local condensates: they are suppressed by powers of $\Lambda_{\mathrm{QCD}} / m v^{2}$ and $\Lambda_{\mathrm{QCD}} / m v$ respectively. If $m v \gg$ $\Lambda_{\mathrm{QCD}} \gg m v^{2}$, the scale $m v$ can be still integrated out perturbatively, giving rise to the Coulombtype potential (2). Non-perturbative contributions to the potential arise when integrating out the scale $\Lambda_{\mathrm{QCD}}$ [3]. We call quasi-Coulombic the systems where the non-perturbative piece of the potential can be considered small with respect to the Coulombic one and treated as a perturbation. Some levels of $t \bar{t}$, the lowest level of $b \bar{b}$ may be considered Coulombic systems 5 , while the $J / \psi$, the $\eta_{c}$ and the short-range hybrids may be considered quasi-Coulombic. The $B_{c}$ may be in a boundary situation, see Table 1 . As it is typical in an effective theory, only the actual calculation may confirm if the initial assumption about the physical system was appropriate.

For all these systems it is relevant to obtain a determination of the energy levels as accurate as possible in perturbation theory.

\section{CALCULATION of QUARKONIUM ENERGIES at $O\left(m \alpha_{s}^{5} \ln \alpha_{s}\right)$}

The perturbative energy levels of quarkonium are known at $O\left(m \alpha_{s}^{4}\right)$ from 12,13]. pNRQCD provides us with a well defined way of calculating

${ }^{5}$ Actually both $b \bar{b}$ and $c \bar{c}$ ground states have been studied in this way 13.17 
the energy levels at higher orders, since the size of each term in the pNRQCD Lagrangian is welldefined. In order to obtain the leading $\operatorname{logs}$ at $O\left(m \alpha_{\mathrm{s}}^{5}\right)$ in the spectrum, $V_{s}$ has to be computed at $O\left(\alpha_{\mathrm{s}}^{4} \ln \right), V_{s}^{(1)}$ at $O\left(\alpha_{\mathrm{s}}^{3} \ln \right), V_{s}^{(2)}$ at $O\left(\alpha_{\mathrm{s}}^{2} \ln \right)$ and $V_{s}^{(3)}$ at $O\left(\alpha_{\mathrm{s}} \ln \right)$. The matching to pNRQCD at $\mathrm{N}^{3} \mathrm{LL}$ accuracy was calculated in [16] and the potentials at the requested accuracy were thus obtained. In Fig.(2) the relevant graphs are shown. In particular from the last graph in pNRQCD (the singlet turns into an octet double-line by emitting an ultrasoft gluon) we extract the dependence of $V_{s}^{(n)}$ on $\mu^{\prime}$.

The total correction to the energy at $O\left(m \alpha_{s}^{5} \ln \alpha_{s}\right)$ is given by the sum of the averaged values of the potentials plus the non-potential effects,

$$
\begin{gathered}
\delta E_{n, l, j}=\delta^{\mathrm{pot}} E_{n, l, j}\left(\mu^{\prime}\right)+\delta^{\mathrm{US}} E_{n, l}\left(\mu^{\prime}\right), \\
\delta E_{n, l, j}^{\mathrm{pot}}\left(\mu^{\prime}\right)=E_{n} \frac{\alpha_{\mathrm{s}}^{3}}{\pi}\left\{\frac { C _ { A } } { 3 } \left[\frac{C_{A}^{2}}{2}+4 C_{A} C_{F} \frac{1}{n(2 l+1)}\right.\right. \\
\left.\quad+2 C_{F}^{2}\left(\frac{8}{n(2 l+1)}-\frac{1}{n^{2}}\right)\right] \ln \frac{\mu^{\prime}}{m \alpha_{\mathrm{s}}} \\
+\frac{C_{F}^{2} \delta_{l 0}}{3 n}\left(8\left[C_{F}-\frac{C_{A}}{2}\right] \ln \frac{\mu^{\prime}}{m \alpha_{\mathrm{s}}}+\left[C_{F}+\frac{17 C_{A}}{2}\right] \ln \alpha_{\mathrm{s}}\right) \\
\left.-\frac{7}{3} \frac{C_{F}^{2} C_{A} \delta_{l 0} \delta_{s 1}}{n} \ln \alpha_{\mathrm{s}}-\frac{\left(1-\delta_{l 0}\right) \delta_{s 1} C_{j, l}}{l(2 l+1)(l+1) n} \frac{C_{F}^{2} C_{A}}{2} \ln \alpha_{\mathrm{s}}\right\},
\end{gathered}
$$

where $E_{n}=-m C_{F}^{2} \alpha_{\mathrm{s}}^{2} /\left(4 n^{2}\right)$ and

$$
C_{j, l}= \begin{cases}-\frac{(l+1)(4 l-1)}{2 l-1} & , j=l-1 \\ -1 & , j=l \\ \frac{l(4 l+5)}{2 l+3} & , j=l+1 .\end{cases}
$$

The $\ln \alpha_{\mathrm{s}}$ appearing in Eq. (何) come from logs of the type $\ln 1 / m r$. Therefore they can be deduced once the dependence on $\ln m$ is known. The $\mu^{\prime}$ dependence of Eq. (4) cancels against contributions from US energies. At the next-to-leading order in the multipole expansion the contribution from these scales reads

$\delta^{\mathrm{US}} E_{n, l}\left(\mu^{\prime}\right)=-i \frac{g^{2}}{3 N_{c}} T_{F} \times$
$\int_{0}^{\infty} d t\left\langle n, l\left|\mathbf{r} e^{i t\left(E_{n}-H_{o}\right)} \mathbf{r}\right| n, l\right\rangle\left\langle\mathbf{E}^{a}(t) \phi(t, 0)_{a b}^{\mathrm{adj}} \mathbf{E}^{b}(0)\right\rangle\left(\mu^{\prime}\right)$, where $H_{o}=\mathbf{p}^{2} / 2 m+V_{o}$.

Different possibilities appear depending on the relative size of $\Lambda_{\mathrm{QCD}}$ with respect to the US scale $m \alpha_{\mathrm{s}}^{2}$. If we consider that $\Lambda_{\mathrm{QCD}} \sim m \alpha_{\mathrm{s}}^{2}$ the gluonic correlator in Eq. (5) cannot be computed using perturbation theory. We are still able to obtain all the $m \alpha_{s}^{5} \ln \left(m \alpha_{s} / m\right)$ and $m \alpha_{s}^{5} \ln \left(m \alpha_{s} / \mu^{\prime}\right)$ contributions where the $\mu^{\prime}$ dependence cancels now against the US contributions that have to be evaluated non-perturbatively.

If we consider that $m \alpha_{\mathrm{s}}^{2} \gg \Lambda_{\mathrm{QCD}}$, Eq. (5) can be computed perturbatively. Being $m \alpha_{\mathrm{s}}^{2}$ the next relevant scale, the effective role of Eq. (5) will be to replace $\mu^{\prime}$ by $m \alpha_{\mathrm{s}}^{2}$ (up to finite pieces that we are neglecting) in Eq. (4). Then Eq. (3) simplifies to

$$
\begin{gathered}
\delta E_{n, l, j}=E_{n} \frac{\alpha_{\mathrm{s}}^{3}}{\pi} \ln \alpha_{\mathrm{s}}\left\{\frac { C _ { A } } { 3 } \left[\frac{C_{A}^{2}}{2}+4 C_{A} C_{F} \frac{1}{n(2 l+1)}\right.\right. \\
\left.+2 C_{F}^{2}\left(\frac{8}{n(2 l+1)}-\frac{1}{n^{2}}\right)\right]+\frac{3 C_{F}^{2} \delta_{l 0}}{n}\left[C_{F}+\frac{C_{A}}{2}\right] \\
\left.-\frac{7}{3} \frac{C_{F}^{2} C_{A} \delta_{l 0} \delta_{s 1}}{n}-\frac{\left(1-\delta_{l 0}\right) \delta_{s 1}}{l(2 l+1)(l+1) n} C_{j, l} \frac{C_{F}^{2} C_{A}}{2}\right\}(6)
\end{gathered}
$$

plus non-perturbative corrections that can be parameterized by local condensates [16, 14, 13] and are of order $\alpha_{s}^{2}\left(\frac{\Lambda_{\mathrm{QCD}}}{m \alpha_{s}}\right)^{2}\left(\frac{\Lambda_{\mathrm{QCD}}}{m \alpha_{s}^{2}}\right)^{2}$ and higher. For the $\Upsilon(1 S)$ and $t \bar{t}$ non-perturbative contributions are expected not to exceed $100 \div 150 \mathrm{MeV}$ and 10 $\mathrm{MeV}$ respectively.

The calculation (6) paves the way to the full $\mathrm{N}^{3} \mathrm{LO}$ order analysis of Coulombic systems and is relevant at least for $t \bar{t}$ production and $\Upsilon$ physics. In the first case it is a step forward reaching a $100 \mathrm{MeV}$ sensitivity on the top quark mass for the $t \bar{t}$ cross section near threshold to be measured at future Linear Colliders [24]. In the second case they improve our knowledge of the $b$ mass 417,25$]$.

In particular, from (6) we can estimate the $\mathrm{N}^{3} \mathrm{LL}$ correction to the energy level of the $\Upsilon(1 S)$ and we find

$$
\begin{aligned}
\delta E_{101}= & \frac{1730}{81 \pi} m_{b} \alpha_{s}^{4}(\mu) \alpha_{s}\left(\mu^{\prime}\right) \ln 1 / \alpha_{s}\left(\mu^{\prime}\right) \\
& \simeq(80 \div 100) \mathrm{MeV},
\end{aligned}
$$

which appears not to be small. Corrections from this $\mathrm{N}^{3} \mathrm{LL}$ terms have been calculated for the $t \bar{t}$ cross section and $\Upsilon(1 S)$ wave function, cf. 18 and [17. They also turn out to be sizeable. 
Large corrections, however, already show up at $\mathrm{NLO}$ and $\mathrm{N}^{2} \mathrm{LO}$ and are responsible for the bad convergence of the perturbative series in terms of the pole mass. This will be discussed in the next section.

\section{RENORMALONS, the POLE MASS and the PERTURBATIVE EXPAN- SION}

The bad convergence of the perturbative expansion (for an explicit example see Sec.7) can be, at least in part, attributed to renormalon contributions. The pole mass, thought an infrared safe quantity |19], has long distance contributions of order $\Lambda_{\mathrm{QCD}}$ [20]. Also the static potential is affected by renormalons (see e.g. [20]). Rephrasing them in the effective field theory language of pNRQCD we can say that the Coulomb potential suffers from IR renormalons ambiguities with the following structure

$\left.V_{s}(r)\right|_{\text {IR ren }}=C_{0}+C_{2} r^{2}+\ldots$

The constant $C_{0} \sim \Lambda_{\mathrm{QCD}}$ is known to be cancelled by the IR pole mass renormalon $\left(\left.2 m_{\text {pole }}\right|_{\text {IR ren }}=-C_{0},[20]\right)$. Several mass definitions appropriate to explicitly realize this renormalon cancellation have been proposed[21]. Among the others, the $1 S$ mass 22 is defined as half of the perturbative contribution to the ${ }^{3} S_{1}$ $q \bar{q}$ mass. Unlike the pole mass, the $1 S$ mass, containing, by construction, half of the total static energy $\left\langle 2 m+V^{\text {Coul }}\right\rangle$, is free of ambiguities of order $\Lambda_{\mathrm{QCD}}$. Taking e.g. the $\Upsilon(1 S)$, the $1 S$ mass is related to the physical $\Upsilon(1 S)$ mass by $E(\Upsilon(1 S))=2 m_{1 S}+\Lambda_{\Upsilon} . \Lambda_{\Upsilon}$ is the poorly known non-perturbative contribution, which is likely, as we said, to be less than $100 \div 150 \mathrm{MeV}$. In the next section we will present an explicit example that shows how, using this mass and thus dealing with quantities that are infrared safe at order $\Lambda_{\mathrm{QCD}}$, the pathologies of the perturbative series, due to the renormalon ambiguities affecting the pole mass, are cured.

It is possible to show that the second infrared renormalon, $C_{2} \simeq \Lambda_{\mathrm{QCD}}^{3}$, of $V_{s}$ cancels against the appropriate pNRQCD UV renormalon in the contribution to the potential originating at NLO in the multipole expansion. What remains is the explicit expression for the operator which absorbs the $C_{2} \sim \Lambda_{\mathrm{QCD}}^{3}$ ambiguity (for details see [3]). An interesting open question is if an explicit renormalon subtraction similar to that one at $O\left(\Lambda_{\mathrm{QCD}}\right)$ can be realized at the subsequent order $O\left(\Lambda_{\mathrm{QCD}}^{3}\right)$. This may be relevant since this renormalon is related to the corrections at $\mathrm{N}^{3} \mathrm{LL}$ discussed in the previous section. Indeed, it is still an open problem whether the largeness of the $\mathrm{N}^{3} \mathrm{LL}$ corrections is an artifact due to our partial knowledge of the contributions at this order, or if it is an artifact due to the fact that the subsequent renormalon cancellation has to be realized at this order or finally if it is a true signal of the breakdown of the perturbative series. To make more definite statements one should know the complete $\mathrm{N}^{3} \mathrm{LO}$ or understand the mechanism of cancellation of the second renormalon.

In the next section I will present a concrete example of the relevance of the mass renormalon cancellation in order to obtain reliable phenomenological predictions.

\section{THE PERTURBATIVE CALCULA- TION of the $B_{c}$ MASS}

We consider the perturbative calculation up to order $m \alpha_{s}^{4}$ of the energy of the $\bar{b} c$ ground state: this will be relevant to a QCD determination of the $B_{c}$ mass if this system is Coulombic or at least quasi-Coulombic. We also assume the $\Upsilon(1 S)$ and the $J / \psi$ to be Coulombic or quasi. The question if these assumptions correspond to the actual systems cannot be settled here. On the other hand there is no a priori reason to rule them out. The results will tell us how good were our initial assumptions.

In order to calculate the $B_{c}$ mass in perturbation theory up to order $\alpha_{\mathrm{s}}^{4}$, we only need to consider the following contributions to the potential: the perturbative static potential at two loops, the $1 / m$ relativistic corrections at one loop, the spinindependent $1 / \mathrm{m}^{2}$ relativistic corrections at tree level and the $1 / \mathrm{m}^{3}$ correction to the kinetic energy. We do not consider $\alpha_{s}^{5} \ln \alpha_{s}$ corrections since the mechanism responsible for this large contributions has not yet been understood. Then, 
we have 23,13

$$
\begin{aligned}
& E\left(B_{c}\right)_{\text {pert }}=m_{b}+m_{c}+E_{0}(\bar{\mu})\left\{1-\frac{\alpha_{\mathrm{s}}(\bar{\mu})}{\pi}\right. \\
& {\left[\beta_{0} l+\frac{4}{3} C_{A}-\frac{11}{6} \beta_{0}\right]+\left(\frac{\alpha_{\mathrm{s}}}{\pi}\right)^{2}\left[\frac{3}{4} \beta_{0}^{2} l^{2}+\left(2 C_{A} \beta_{0}\right.\right.} \\
& \left.-\frac{9}{4} \beta_{0}^{2}-\frac{\beta_{1}}{4}\right) l-\pi^{2} C_{F}^{2}\left(\frac{1}{m_{b}^{2}}+\frac{1}{m_{c}^{2}}-\frac{6}{m_{b} m_{c}}\right) m_{\mathrm{red}}^{2} \\
& +\frac{5}{4} \pi^{2} C_{F}^{2}\left(\frac{1}{m_{b}^{3}}+\frac{1}{m_{c}^{3}}\right) m_{\mathrm{red}}^{3}+\pi^{2} C_{F} C_{A}+\frac{4}{9} C_{A}^{2}- \\
& \left.\left.\frac{17}{9} C_{A} \beta_{0}+\left(\frac{181}{144}+\frac{1}{2} \zeta(3)+\frac{\pi^{2}}{24}\right) \beta_{0}^{2}+\frac{\beta_{1}}{4}+\frac{c}{8}\right]\right\},
\end{aligned}
$$
being $m_{\text {red }}=m_{b} m_{c} /\left(m_{b}+m_{c}\right), \quad l=$ $\ln \left(2 C_{F} \alpha_{\mathrm{s}} m_{\text {red }} / \bar{\mu}\right), E_{0}(\bar{\mu})=-m_{\text {red }}\left(C_{F} \alpha_{\mathrm{s}}(\bar{\mu})\right)^{2} / 2$ and $\bar{\mu}$ the scale around which we expand $\alpha_{s}(r)$.

If we use here the pole masses $m_{b}=5 \mathrm{GeV}$, $m_{c}=1.8 \mathrm{GeV}$ and $\bar{\mu}=1.6 \mathrm{GeV}$, then we obtain $E\left(B_{c}\right)_{\text {pert }} \simeq 6149 \mathrm{MeV} \simeq 6800-115-183-353$ $\mathrm{MeV}$, where the second, third and fourth figures are the corrections of order $\alpha_{\mathrm{s}}^{2}, \alpha_{\mathrm{s}}^{3}$ and $\alpha_{\mathrm{s}}^{4}$ respectively. The series turns out to be very badly convergent. This reflects also in a strong dependence on the normalization scale $\bar{\mu}$ : at $\bar{\mu}=1.2$ $\mathrm{GeV}$ we would get $E\left(B_{c}\right)_{\text {pert }} \simeq 5860 \mathrm{MeV}$, while at $\bar{\mu}=2.0 \mathrm{GeV}$ we would get $E\left(B_{c}\right)_{\text {pert }} \simeq 6279$ $\mathrm{MeV}$. The non-convergence of the perturbative series (9) signals the fact that large $\beta_{0}$ contributions (coming from the static potential renormalon) are not summed up and cancelled against the pole masses. In order to obtain a wellbehaved perturbative expansion, we use, now, the so-called $1 S$ mass. We consider the perturbative contribution (up to order $\alpha_{s}^{4}$ ) of the ${ }^{3} S_{1}$ levels of charmonium and bottomonium:

$$
E(J / \psi)_{\mathrm{pert}}=f\left(m_{c}\right) ; \quad E(\Upsilon(1 S))_{\mathrm{pert}}=f\left(m_{b}\right),
$$

which are respectively a function of the $c$ and the $b$ pole mass and can be read off from Eq. (9) in the equal-mass case, adding to it the spin-spin interaction energy: $m\left(C_{F} \alpha_{\mathrm{s}}\right)^{4} / 3$. We invert these relations in order to obtain the pole masses as a formal perturbative expansion depending on the $1 S$ masses. Finally, we insert the expressions $m_{c}=$ $f^{-1}\left(E(J / \psi)_{\text {pert }}\right)$ and $m_{b}=f^{-1}\left(E(\Upsilon(1 S))_{\text {pert }}\right)$ in Eq. (9). At this point we have the perturbative mass of the $B_{c}$ as a function of the $J / \psi$ and $\Upsilon(1 S)$

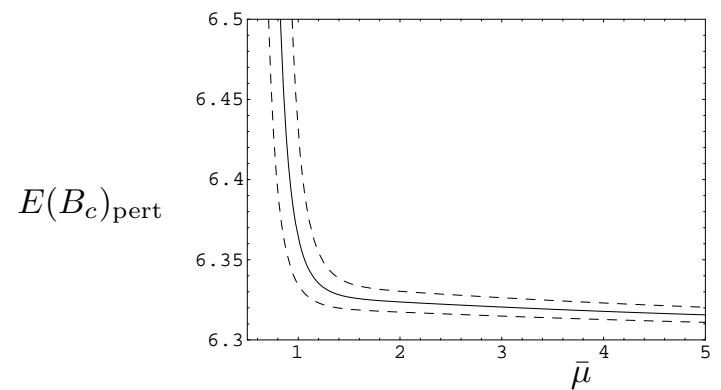

Figure 3. $E\left(B_{c}\right)_{\text {pert }}$ as a function of $\bar{\mu}$ at $\Lambda \frac{N_{f}=3}{\mathrm{MS}}=300 \mathrm{MeV}$ (continuous line). The dashed lines refer to $\Lambda_{\overline{\mathrm{MS}}}^{N_{f}=3}=250,350 \mathrm{MeV}$ respectively.

perturbative masses. If we identify the perturbative masses $E(J / \psi)_{\text {pert }}, E(\Upsilon(1 S))_{\text {pert }}$ with the physical ones, then the expansion (9) depends only on the scale $\bar{\mu}$. The perturbative series turns out to be reliable for values of $\bar{\mu}$ bigger than $(1.2 \div 1.3) \mathrm{GeV}$ and lower than $(2.6 \div 2.8) \mathrm{GeV}$. For instance, $E\left(B_{c}\right)_{\text {pert }}=6278.5+35+6.5+5.5$ $\mathrm{MeV}$ at the scale $\bar{\mu}=1.6 \mathrm{GeV}$. Therefore, we obtain now a better convergence of the perturbative expansion and a stable determination of the perturbative mass of the $B_{c}$. This fact seems to support the $B_{c}$ being indeed a Coulombic or quasi-Coulombic system. By varying $\bar{\mu}$ from 1.2 $\mathrm{GeV}$ to $2.0 \mathrm{GeV}$ and $\Lambda_{\overline{\mathrm{MS}}}^{N_{f}=3}$ from $250 \mathrm{MeV}$ to 350 $\mathrm{MeV}$ and by calculating the maximum variation of $E\left(B_{c}\right)_{\text {pert }}$ in the given range of parameters, we get as our final result

$E\left(B_{c}\right)_{\text {pert }}=6326_{-9}^{+29} \mathrm{MeV}$.

As a consequence of the now obtained good behaviour of the perturbative series in the considered range of parameters, the result appears stable with respect to variations of $\bar{\mu}$ (see Fig. 3) and, therefore, reliable from the perturbative point of view. It represents a rather clean prediction of the lowest mass of the $B_{c}$. Notice that all the existing predictions are based either on potential models or on lattice evaluation (with still large errors).

Non-perturbative contributions have not been taken into account so far. They affect the identification of the perturbative masses $E\left(B_{c}\right)_{\text {pert }}$, $E(\Upsilon(1 S))_{\text {pert }}, E(J / \psi)_{\text {pert }}$, with the correspond- 
ing physical ones through Eq.(9). Let us call these non-perturbative contributions $\Lambda_{B_{c}}, \Lambda_{\Upsilon}$ and $\Lambda_{J / \psi}$ respectively. As discussed before, they can be of potential or non-potential nature. In the latter case they can be encoded into nonlocal condensates or into local condensates. Nonperturbative contributions affect the identification with the physical $B_{c}$ mass roughly by an amount $\simeq-\Lambda_{J / \psi} / 2-\Lambda_{\Upsilon} / 2+\Lambda_{B_{c}}$. Assuming $\left|\Lambda_{J / \psi}\right| \leq 300 \mathrm{MeV},\left|\Lambda_{\Upsilon}\right| \leq 100 \mathrm{MeV}$ and $\Lambda_{\Upsilon} \leq$ $\Lambda_{B_{c}} \leq \Lambda_{J / \psi}$, the identification of our result (10) with the physical $B_{c}$ mass may, in principle, be affected by uncertainties, due to the unknown nonperturbative contributions, as big as $\pm 200 \mathrm{MeV}$. However, the different non-perturbative contributions $\Lambda$ are correlated, so that we expect, indeed, smaller uncertainties. If we assume, for instance, $\Lambda_{\Upsilon}$ and $\Lambda_{J / \psi}$ to have the same sign, which seems to be quite reasonable, then the above uncertainty reduces to $\pm 100 \mathrm{MeV}$. This would confirm, indeed, that the effect of the non-perturbative contributions on the result of Eq. (10) is not too large.

\section{CONCLUSION and OUTLOOK}

We have shown that for heavy quark bound systems in the situation $\Lambda_{\mathrm{QCD}} \lesssim m v^{2}$, the energy levels turn out to be calculable in perturbation theory plus local or non-local condensates. pNRQCD provides us with the appropriate tool to calculate these energy levels at the desired order in perturbation theory. The obtained $\mathrm{N}^{3} \mathrm{LL}$ contributions turn out to be sizeable. A complete calculation at $\mathrm{N}^{3} \mathrm{LO}$ is required to settle this issue 26]. For the practical use of the obtained perturbative series, renormalon cancellation mechanisms seem to be important. This may be relevant also at order $\alpha_{s}^{5} \ln \alpha_{s}$. In particular, I have discussed how a renormalon-free definition of the quark masses improves considerably the behaviour of the perturbative series on the concrete example of the $B_{c}$ mass calculation.

\section{Acknowledgments}

I thank the Organizers for invitation and support and the Humboldt foundation for support.I thank A. Vairo for reading the manuscript and comments.

S. Peris (U.A. Barcelona): In your Schrödinger equation what definition of the quark mass are you supposed to use?

N. Brambilla: The pole mass. Any other definition of the mass that does not change the power counting can be used, cf. Secs. 6 and 7.

\section{REFERENCES}

1. N. Brambilla and A. Vairo, hep-ph/9904330; G. S. Bali, hep-ph/0001312; N. Brambilla and A. Vairo, Phys. Rev. D55, 3974 (1997) hep$\mathrm{ph} / 9606344$.

2. A. Pineda and J. Soto, Nucl. Phys. Proc. Suppl. 64, 428 (1998) hep-ph/9707481.

3. N. Brambilla, A. Pineda, J. Soto and A. Vairo, Nucl. Phys. B566, 275 (2000) hepph/9907240|; N. Brambilla, hep-ph/0008279.

4. A. Vairo, hep-ph/0009146.

5. N. Brambilla, A. Pineda, J. Soto and A. Vairo, hep-ph/0002250; A. Pineda and A. Vairo, hep-ph/0009145.

6. T. Appelquist, M. Dine and I. J. Muzinich, Phys. Rev. D17, 2074 (1978); L. S. Brown and W. I. Weisberger, Phys. Rev. D20, 3239 (1979).

7. N. Brambilla, A. Pineda, J. Soto and A. Vairo, Phys. Rev. D60, 091502 (1999) hep-ph/9903355.

8. M. Beneke, A. Signer and V. A. Smirnov, Phys. Lett. B454, 137 (1999) hepph/9903260]; M. E. Luke, A. V. Manohar and I. Z. Rothstein, Phys. Rev. D61, 074025 (2000) hep-ph/9910209 and refs. therein.

9. W. E. Caswell and G. P. Lepage, Phys. Lett. B167, 437 (1986); G. T. Bodwin, E. Braaten and G. P. Lepage, Phys. Rev. D51, 1125 (1995) hep-ph/9407339.

10. N. Brambilla, Nucl. Phys. Proc. Suppl. 86, 389 (2000) hep-ph/9909211; A. Pineda, Nucl. Phys. Proc. Suppl. 86, 517 (2000) hepph/9910239; A. Vairo, Nucl. Phys. Proc. Suppl. 86, 521 (2000) hep-ph/9909440.

11. Y. Schroder, Phys. Lett. B447, 321 (1999) hep-ph/9812205; M. Peter, Phys. Rev. Lett. 
78, 602 (1997) hep-ph/9610209; A. Billoire, Phys. Lett. B92, 343 (1980).

12. S. N. Gupta and S. F. Radford, Phys. Rev. D24, 2309 (1981).

13. S. Titard and F. J. Yndurain, Phys. Rev. D49, 6007 (1994) hep-ph/9310236; A. Pineda and F. J. Yndurain, Phys. Rev. D61, 077505 (2000) hep-ph/9812371.

14. M. B. Voloshin, Nucl. Phys. B154, 365 (1979); H. Leutwyler, Phys. Lett. B98, 447 (1981).

15. N. Brambilla and A. Vairo, hep-ph/0004192.

16. N. Brambilla, A. Pineda, J. Soto and A. Vairo, Phys. Lett. B470, 215 (1999) hepph/9910238; B. A. Kniehl and A. A. Penin, Nucl. Phys. B563, 200 (1999) hepph/9907489.

17. F. J. Yndurain, hep-ph/0008007; F. J. Yndurain, hep-ph/0002237.

18. A. Penin, these proceedings; B. A. Kniehl and A. A. Penin, Nucl. Phys. B577, 197 (2000) hep-ph/9911414.

19. A. S. Kronfeld, Phys. Rev. D58, 051501 (1998) hep-ph/9805215.

20. M. Beneke, Phys. Lett. B434, 115 (1998) hep-ph/9804241. A. H. Hoang, M. C. Smith, T. Stelzer and S. Willenbrock, Phys. Rev. D59, 114014 (1999) hep-ph/9804227.

21. A. H. Hoang and T. Teubner, Phys. Rev. D60, 114027 (1999) hep-ph/9904468; I. Bigi, M. Shifman and N. Uraltsev, Ann. Rev. Nucl. Part. Sci. 47, 591 (1997) hep$\mathrm{ph} / 9703290$; O. Yakovlev and S. Groote, hep-ph/0009014.

22. A. H. Hoang, Z. Ligeti and A. V. Manohar, Phys. Rev. Lett. 82, 277 (1999) hepph/9809423; A. H. Hoang, Z. Ligeti and A. V. Manohar, Phys. Rev. D59, 074017 (1999) hep-ph/9811239.

23. N. Brambilla and A. Vairo, hep-ph/0002075.

24. A. H. Hoang et al., Eur. Phys. J. direct C3, 1 (2000) hep-ph/0001286.

25. A. H. Hoang, hep-ph/0008102.

26. Y. Kiyo and Y. Sumino, hep-ph/0007251. 